\title{
UNION OF CONVEX HILBERT CUBES
}

\author{
J. QUINN AND RAYMOND Y. T. WONG ${ }^{1}$
}

\begin{abstract}
We show that the finite union of Keller cubes in a Hilbert space is homeomorphic to the Hilbert cube provided every subcollection intersects in a Hilbert cube.
\end{abstract}

1. Introduction. In this paper, we establish the following result:

If $K_{1}, K_{2}, \ldots, K_{n}$ is a collection of convex Hilbert cubes contained in $l_{2}$ such that every subcollection intersects in a Hilbert cube, then $\cup_{i=1}^{n} K_{i}$ is homeomorphic to the Hilbert cube.

This result, for $n=2$, answers a question stated in [AK, LS4, p. 166]. Because of its potential usefulness in studying hyperspaces of compact convex sets the same question was also posed in [NQS $\mathbf{N}_{2}$.

The more general question (see $\left[\mathbf{A}_{1}\right]$ and $[\mathbf{A B}]$ ) of whether the union of two Hilbert cubes which intersect in a Hilbert cube is a Hilbert cube has been recently solved in the negative by $\mathbf{R}$. Sher [S]. Results in a positive direction have been obtained by R. D. Anderson [ $\left.\mathbf{W}_{\mathbf{1}}\right]$, N. Kroonenberg and R. Wong $[\mathbf{K W}]$ and $\mathrm{M}$. Handel $\left[\mathbf{H}_{2}\right]$. It should be mentioned that the results of this paper are easy consequences of the work in $\left[\mathbf{H}_{2}\right]$ and $[K]$.

The first author would like to thank B. J. Ball who read a preliminary draft of this manuscript and made several very helpful comments.

2. Some preliminaries. The reader is referred to $[\mathbf{K U}]$ as a general reference for results and definitions in the area of topology. In what follows, we will denote by $Q$ the Hilbert cube $Q=\prod_{i=1}^{\infty}[-1,1]_{i}$. A Hilbert cube is a space homeomorphic $(\cong)$ to $Q$. By a Keller cube we mean a Hilbert cube which is a compact convex subset of Hilbert space $l_{2}$. The reader should note that, since every compact subset of a metrizable locally convex topological vector space can be affinely embedded in $l_{2}$, the results of this paper will be true in such an "apparently" more general setting. A set $A \subset Q$ is said to be a $Z$-set if for any nonempty and homotopically trivial open subset $U$ of $Q, U-A$ is nonempty and homotopically trivial. If $Y$ is a metric space, a set $A \subset Y$ is said to be $k-L c c$ ( $k$ is a nonnegative integer) embedded in $Y$ if for every $p \in A$ and $\varepsilon>0$ there exists a $\delta>0$ such that any $f: S^{k} \rightarrow B(\delta, p)-A$ can

Presented to the Society, January 24, 1976; received by the editors May 24, 1976 and, in revised form, September 4, 1976.

AMS (MOS) subject classifications (1970). Primary 57A20, 52-00; Secondary 54B20.

$K e y$ words and phrases. Keller cubes, Hilbert cubes, $Z$-sets, f.d. CAP sets, $Q$-manifolds.

${ }^{1}$ The second author is partially supported by NSF Grant MPS75-08334. 
be extended to a mapping $\bar{f}: B^{k+1} \rightarrow B(\varepsilon, p)-A$, where $B(\varepsilon, p)=\{y \in Y$ : $d(y, p)<\varepsilon\}$. If $X \subset l_{2}$ then the linear span of $X$, denoted $\operatorname{Sp}(X)$, is the set of all $y \in l_{2}$ such that $y$ is collinear with at least two distinct points of $X$. We will denote the closure of a set $N$ by $\operatorname{cl}(N)$ and the convex hull of $N$ by $\operatorname{co}(N)$. A metric space $X$ is said to be $L C^{n}$ if, for every $0 \leqslant k \leqslant n$, for every $p \in X$ and for every $\varepsilon>0$ there exists a $\delta>0$ such that any mapping of $S^{k}$ into $B(\delta, p)$ can be extended to a mapping of $B^{k+1}$ into $B(\varepsilon, p)$.

A set $M=\cup_{i=1}^{\infty} M_{i}$ is said to be a (f.d.) CAP set for $Q$ if, for each $i, M_{i}$ is a (f.d.) $Z$-set in $Q, M_{i} \subset M_{i+1}$ and, given $n_{0}, \varepsilon>0$ and the (f.d.) $Z$-set $Y \subset Q$, there exist an $n_{1}$ and a space homeomorphism $h$ of $Q$ onto $Q$ such that $h \mid M_{n_{0}}=\mathrm{id}, h(Y) \subset M_{n_{1}}$ and $d\left(h, \mathrm{id}_{Q}\right)<\varepsilon$. The concept of a CAP set was introduced by R. D. Anderson (cf. $\left[\mathbf{A}_{2}\right]$ ). (Note. f.d. is brief for "finite dimensional".)

In his paper $\left[\mathbf{H}_{2}\right]$ on sums of Hilbert cubes, Michael Handel has all but stated the following result:

LEMMA A (M. HANDEL). Let $Q_{1} \cong Q_{2} \cong Q_{1} \cap Q_{2}=Q_{3} \cong Q$. If $Q_{2}$ has an f.d. $C A P$ set $M=\cup_{i=1}^{\infty} M_{i}$ such that $M_{i} \cap Q_{1}$ is a Z-set in $Q_{1}$, for each $i$, then $Q_{1} \cup Q_{2} \cong Q$

\section{The main results.}

(3.1) LemMA. If $K=\cup_{i=1}^{n} K_{i}$ is a Hilbert cube in $l_{2}$ such that, for each $i, K_{i}$ is a Keller cube and such that $\cap_{i=1}^{n} K_{i} \cong Q$, then any closed set of finite dimensional linear span lying in $K$ is a $Z$-set in $K$.

Proof. Let $P \subset K$ be a closed set of finite linear span. Nelly Kroonenberg has shown [K] that a finite dimensional closed subset of $Q$ which is $1-L c c$ embedded in $Q$ is a $Z$-set in $Q$. We will show that $P$ is $1-L c c$ embedded in $K$. Let $p \in P$ and $\varepsilon>0$ be given. Let $\delta>0$ be chosen so that if $K_{j}$ is such that $p \notin K_{j}$ then $B(\delta, p) \cap K_{j}=\varnothing$. Let $f: S^{1} \rightarrow B^{*}(\delta, p)-P$, where $B^{*}(\delta, p)=$ $B(\delta, p) \cap K$. We want to show there is a homotopy $h: S^{1} \times I \rightarrow K$ such that $h(s, 0)=f(s), h\left(S^{1} \times 1\right)$ is a polyhedron and $h\left(S^{1} \times t\right) \subset B^{*}(\delta, p)-P$ for all $0 \leqslant t \leqslant 1$. We will show that

(*) for every $\gamma>0$, there exists a triangulation $T=\left\{v_{1}, v_{2}, \ldots, v_{m}\right\}$ of $S^{1}$ such that $\operatorname{diam}\left(f\left(v_{i}, v_{i+1}\right)\right)<\gamma / 2$ and for each $i$ there exists a $K_{j}$ such that $\left\{f\left(v_{i}\right), f\left(v_{i+1}\right)\right\} \subset K_{j}$.

Under these circumstances, we can conclude that any mapping $\boldsymbol{g}$ of $S^{\mathbf{1}}$ onto $\cup_{i=1}^{m}\left[f\left(v_{i}\right), f\left(v_{i+1}\right)\right]$ such that $g\left(v_{i}, v_{i+1}\right)=\left[f\left(v_{i}\right), f\left(v_{i+1}\right)\right]$ and $g\left(v_{i}\right)=$ $f\left(v_{i}\right)$ for $i=1,2, \ldots, m$ (note $v_{n+1}=v_{1}$ ) satisfies the property that $d(g, f)$ $<\gamma$. Since $B^{*}(\delta, p)$ is (in particular) an $L C^{1}$ space, the above is enough to guarantee the existence of the desired homotopy $h$. To see that (*) holds, let $\gamma>0$ be given and let $T=\left\{v_{1}, v_{2}, \ldots, v_{m}\right\}$ be a triangulation of $S^{1}$ such that $\operatorname{diam}\left(f\left(\left(v_{i}, v_{i+1}\right)\right)\right)<\gamma / 2$. If, for every $i,\left\{f\left(v_{i}\right), f\left(v_{i+1}\right)\right\} \subset K_{j}$, for some $j$, we are done. Suppose there does not exist a $K_{j}$ such that $\left\{f\left(v_{i}\right), f\left(v_{i+1}\right)\right\} \subset$ $K_{j}$. Let $u_{1}=\sup \left\{u \in\left(v_{i}, v_{i+1}\right): f(u)\right.$ and $f\left(v_{i}\right)$ lie in some common $\left.K_{j}\right\}$. Note 
$u_{1} \neq v_{i}$. If $f\left(u_{1}\right)$ and $f\left(v_{i+1}\right)$ belong to $K_{j}$ for some $j$, then we merely refine $T$ by the addition of the vertex $u_{1}$ between $v_{i}$ and $v_{i+1}$. If $f\left(u_{1}\right)$ and $f\left(v_{i+1}\right)$ do not belong to a common $K_{j}$, then let $u_{2}=\sup \left\{u: u \in\left(u_{1}, v_{i+1}\right)\right.$ and $f(u)$ and $f\left(u_{1}\right)$ belong to a common $K_{j}$. Proceeding in this fashion, we eventually obtain $u_{1}, u_{2}, \ldots, u_{s}$ such that, for $k<s, f\left(u_{k}\right)$ and $f\left(v_{i+1}\right)$ do not belong to a common $K_{j},\left\{f\left(u_{k-1}\right), f\left(u_{k}\right)\right\}$ is contained in some $K_{j}, f\left(u_{i}\right)$ and $f\left(u_{k+1}\right)$ do not belong to a common $K_{j}$, for $1 \leqslant i \leqslant k-1$, and $f\left(u_{s}\right)$ and $f\left(v_{i+1}\right)$ belong to a common $K_{j}$. We now refine the triangulation $T$ by the addition of the vertices $u_{1}, u_{2}, \ldots, u_{s}$ between $v_{i}$ and $v_{i+1}$. We can now clearly conclude the validity of (*). So, let $h: S^{1} \times I \rightarrow K$ be a homotopy such that $h(s, 0)=f(x)$, $h\left(S^{1} \times 1\right)$ is a polyhedron, and

$$
h\left(S^{1} \times I\right) \subset B^{*}(\delta, p)-P .
$$

Let $g: S^{1} \times I \rightarrow B(\delta, p)$ be defined by $g(s, t)=(1-t) h(s, 1)+t p$. Let $q \in \cap_{i=1}^{n} K_{i}$ be such that $q \notin \operatorname{Sp}\left(g\left(S^{1} \times I\right) \cup P\right)$. Let $l>0$ be chosen so that, for $r \in[0, l]$ and $(s, t) \in S^{1} \times I,(1-r) g(s, t)+r q \in B^{*}(\delta, p)$. Define $H: S^{1} \times[0, l+2] \rightarrow B^{*}(\delta, p)-P$ by

$$
H(s, t)= \begin{cases}h(s, t) & \text { for } t \in[0,1], \\ (2-t) g(s, 0)+(t-1) q & \text { for } t \in[1,1+l], \\ (1-l) g(s, t-(1+l))+l q & \text { for } t \in[1+l, 2+l] .\end{cases}
$$

The lemma is proved.

(3.2) TheOREM. Let $K_{1}, K_{2}, \ldots, K_{n}$ be a collection of Keller cubes such that every subcollection of the $\left\{K_{i}\right\}_{i=1}^{n}$ intersects in a Hilbert cube. Then $\cup_{i=1}^{n} K_{i} \cong$ $Q$.

Proof. The result is true for $n=1$. Assume it is true for $k \leqslant n$ and suppose $K_{1}, K_{2}, \ldots, K_{n}, K_{n+1}$ is a collection of Keller cubes such that the hypothesis of the lemma is satisfied. Let $M=\cup_{i=1}^{\infty} M_{i}$ be an f.d. CAP set for $K_{n+1}$ such that $M_{i}$ is convex for each $i$. That such an $M$ can be found follows from Proposition 5.1 of [BP, p. 162]. By the induction hypothesis, $\bigcup_{i=1}^{n} K_{i} \cong Q$ and $K_{n+1} \cap\left(\cup_{i=1}^{n} K_{i}\right)=\bigcup_{i=1}^{n}\left(K_{n+1} \cap K_{i}\right) \cong Q$. By Handel's result (Lemma A) it suffices to see that $M_{i} \cap\left(\cup_{j=1}^{n} K_{j}\right)$ is a $Z$-set in $\bigcup_{j=1}^{n} K_{j}$. But, $M_{i} \cap\left(\cup_{j=1}^{n} K_{j}\right)$ is a finite sum of finite dimensional convex sets. By (3.1), we have that $M_{i} \cap\left(\cup_{i=1}^{n} K_{i}\right)$ is a $Z$-set in $\bigcup_{i=1}^{n} K_{i}$. The lemma is proved.

(3.3) Corollary. Let $K_{1}, K_{2}, \ldots, K_{n}$ be a collection of Keller cubes such that if $\left\{i_{1}, i_{2}, \ldots, i_{k}\right\} \subset\{1,2, \ldots, n\}$ then either $\cap_{j=1}^{k} K_{i_{j}}=\varnothing$ or $\cap_{j=1}^{k} K_{i j} \cong$ $Q$. Then, $\cup_{i=1}^{n} K_{i}$ is a $Q$-manifold.

Proof. Let $q \in \cup_{i=1}^{n} K_{i}$ and let $i_{1}, i_{2}, \ldots, i_{k}$ be the maximal set of indices such that $q \in \cap_{j=1}^{k} K_{i,}$. Then $\cup_{j=1}^{k} K_{i,}$ is a neighborhood of $q$ and $Q \cong$ $\cup_{j=1}^{k} K_{i}$ by (3.2). The theorem is proved.

T. A. Chapman has proved (see $\left[\mathbf{C}_{\mathbf{1}}\right]$ or $\left[\mathbf{C}_{\mathbf{2}}\right]$ ) that a contractible compact $Q$-manifold is homeomorphic to $Q$. We thus have the following 
(3.4) Corollary. Let $K_{1}, K_{2}, \ldots, K_{n}$ be a collection of Keller cubes such that if $\left\{i_{1}, i_{2}, \ldots, i_{k}\right\} \subset\{1,2, \ldots, n\}$ then either $\cap_{j=1}^{k} K_{i_{j}}=\varnothing$ or $\cap_{j=1}^{k} K_{i_{j}} \cong$ $Q$. Then $\cup_{i=1}^{n} K_{i} \cong Q$ if and only if $\cup_{i=1}^{n} K_{i}$ is contractible.

This next corollary is a restatement of (3.2) for $n=2$.

(3.5) COROLlaRY. If $K_{1} \cong K_{2} \cong K_{1} \cap K_{2} \cong Q$ are Keller cubes then $K_{1} \cup$ $K_{2} \cong Q$.

(3.6) Remark. If $P$ is a polyhedron in $E^{n}$, then we can view $P \times Q$ as a subset of $E^{n} \times l_{2}$. As such, $P \times Q$ is clearly a finite union of convex Hilbert cubes which have the proper intersection properties. Recalling that the results of this paper hold just as well in this setting, we have by (3.4) that $P \times Q$ is locally homeomorphic to $Q$. This result can now be used to give another proof of the result of J. E. West (see Corollary 5.2 of $\left[\mathbf{W}_{2}\right]$ ), which states that if $X$ is a space which can be triangulated by a locally finite simplicial complex, then the product of $X$ with $Q$ is locally homeomorphic to $Q$.

4. Some discussion. It is reasonable to ask whether the results of the preceding section can be extended to cover situations with less stringent intersection requirements than those of (3.2) or (3.3). In this section we will give a counterexample. In fact, we will give three examples. The first example shows that (in a nontrivial sense) at least sometimes the union of three Keller cubes can be homeomorphic to $Q$ even when the intersection of two of them is just a 2-cell. The second example is a nontrivial example of four Keller cubes which pairwise intersect in Keller cubes, whose union is homeomorphic to $Q$ but whose total intersection is a 2-cell. The last example is of three Keller cubes which pairwise intersect in Keller cubes and whose total intersection is a 2-cell (or $n$-cell), and the union of the Keller cubes is not homeomorphic to $Q$. The argument of the last example is suggested to us by the referee to whom we acknowledge our thanks.

If $X$ is a subset of a Banach space then by $\operatorname{cc}(X)$ (the cc-hyperspace of $X$ ) is meant the space of compact convex subsets of $X$ with the Hausdorff metric (see [NQS $\mathbf{N}_{1}$ ). Using the techniques of $\S 2$ of $\left[\mathbf{N Q S}_{3}\right]$ it can be seen that if $\left\{X_{i}\right.$ : $i=1,2, \ldots, n\}$ is a collection of compact subsets of a Banach space then $\cup_{i=1}^{n} \operatorname{cc}\left(X_{i}\right)$ can be affinely embedded into $l_{2}$. All of the examples which follow are obtained by taking unions of cc-hyperspaces of compact convex subsets of $R^{3}$.

(4.1) ExAMPLE. In $R^{2}$ let

$$
\begin{aligned}
& Y_{1}=\operatorname{co}(\{(0,0),(0,1),(-1,1),(-1,0)\}), \\
& Y_{2}=\operatorname{co}(\{(0,0),(-2,0)\}), \text { and } \\
& Y_{3}=\operatorname{co}(\{(0,0),(0,2)\}) .
\end{aligned}
$$

In $R^{3}$ let $X_{i}=Y_{i} \times[0,1]$ and let $K_{i}=\operatorname{cc}\left(X_{i}\right)$ for $i=1,2,3$. It follows from Theorem (2.2) of $\left[\mathrm{NQS}_{3}\right.$ ] (see also [NQS $]$ ) that $K_{1}, K_{2}, K_{3}, K_{1} \cap K_{2}$ and $K_{1} \cap K_{3}$ are all homeomorphic to $Q$. Since $K_{2} \cap K_{3}=K_{1} \cap K_{2} \cap K_{3}=$ $\operatorname{cc}\left(X_{1} \cap X_{2} \cap X_{3}\right)$ is the cc-hyperspace of an arc, we have that $K_{2} \cap K_{3}=K_{1}$ 
$\cap K_{2} \cap K_{3}$ is a 2-cell. We wish to show that $K=\cup_{i=1}^{3} K_{i} \simeq Q$. But $K_{1} \cap K_{2}$ $\cong Q$ and $K_{1}, K_{2}$ are Keller cubes. Thus, $K_{1} \cup K_{2} \cong Q$ by (3.5). Since $K_{3} \cap\left(K_{1} \cup K_{2}\right)=K_{3} \cap K_{1} \cong Q$ since $K_{1} \cap K_{3}$ is a $Z$-set in $K_{1} \cup K_{2}$ (see Example (4.6) of $\left.\left[\mathrm{NQS}_{3}\right]\right)$ we have, by Theorem 1 of $\left[\mathbf{H}_{2}\right]$, that $K_{3} \cup\left(K_{1} \cup\right.$ $\left.K_{2}\right) \cong Q$. We have shown that $\cup_{i=1}^{3} K_{i} \cong Q$.

A trivial example of four Keller cubes which pairwise intersect in Keller cubes, have total intersection a 2-cell and whose union is homeomorphic to $Q$ can be obtained from any example of three Keller cubes with the same intersection properties by taking the fourth Keller cube to be one which contains the union of the other three. We prefer to have an example for which no Keller cube contains any of the others.

(4.2) EXAMPLE. In $R^{1}$ let

$$
\begin{aligned}
& Y_{1}=\operatorname{co}(\{(0,0),(0,1),(-1,1),(-1,0)\}), \\
& Y_{2}=\operatorname{co}(\{(0,0),(1,0),(1,1),(0,1)\}), \\
& Y_{3}=\operatorname{co}\left(\left\{\left(-1, \frac{1}{2}\right),(-1,0),(1,0),\left(1, \frac{1}{2}\right)\right\}\right), \text { and } \\
& Y_{4}=\operatorname{co}(\{(-1,0),(-1,-1),(1,-1),(1,0)\}) .
\end{aligned}
$$

In $R^{3}$ let $X_{i}=Y_{i} \times[0,1]$ and let $K_{i}=\operatorname{cc}\left(Y_{i}\right)$ for $i=1,2,3,4$. It follows from Theorem (2.2) of [NQS $\mathrm{NQ}_{3}$ that $K_{1}, K_{2}, K_{3}, K_{4}, K_{1} \cap K_{2}, K_{1} \cap K_{3}, K_{1} \cap$ $K_{4}, K_{2} \cap K_{3}, K_{2} \cap K_{4}$ and $K_{3} \cap K_{4}$ are all homeomorphic to $Q$. As in (4.1), $\cap_{i=1}^{4} K_{i}$ is the cc-hyperspace of an arc and is thus a 2-cell. To see that $\cup_{i=1}^{4} K_{i} \cong Q$, we will first consider $\cup_{i=1}^{3} K_{i}$. Since $\cap_{i=1}^{3} K_{i} \cong Q$, we have by (3.2) that $\cup_{i=1}^{3} K_{i} \cong Q$. Now, $K_{4} \cap\left(\cup_{i=1}^{3} K_{i}\right)=K_{4} \cap K_{3} \cong Q$ and $K_{4} \cap$ $\left(\cup_{i=1}^{3} K_{i}\right)$ is a $Z$-set in $K_{4}$ (see Example (4.6) of [NQS $\mathbf{S}_{3}$ ). We thus have that $K_{4} \cup\left(\cup_{i=1}^{3} K_{i}\right)=\cup_{i=1}^{4} K_{i} \cong Q$.

This next example is of three Keller cubes which pairwise intersect in Keller cubes and have total intersection of a 2-cell. Their union, however, is not homeomorphic to $Q$.

(4.3) EXAMPLE. In $R^{2}$ let

$$
\begin{aligned}
& Y_{1}=\operatorname{co}(\{(0,0),(1,0),(2,-1),(0,-1)\}), \\
& Y_{2}=\operatorname{co}(\{(0,0),(1,1),(2,1),(0,1)\}), \text { and } \\
& Y_{3}=\operatorname{co}(\{(1,0),(2,1),(2,-1)\}) .
\end{aligned}
$$

In $R^{3}$ let $X_{i}=Y_{i} \times[0,1]$ for $i=1,2,3$. Let $K_{i}=\operatorname{cc}\left(X_{i}\right)$ for $i=1,2,3$. We have that $K_{i_{1}} \cap K_{i_{2}} \cong Q$ for $i_{1} \neq i_{2}$ and $\cap_{i=1}^{3} K_{i} \cong I^{2}$. It can be easily shown, using Anderson's $Z$-set homeomorphic extension theorem, that $K_{1} \cup K_{2} \cup$ $K_{3}$ is homeomorphic to $\operatorname{Cone}\left(S^{1} \times Q\right) \times I^{2}$, which is not homeomorphic to $Q$. We argue as follows: Let $\left(v_{1}, v_{2}\right) \in \operatorname{Cone}\left(S^{1} \times Q\right) \times I^{2}$, where $v_{1}$ is the cone point and $v_{2}$ is any interior point of $I^{2}$. If $\operatorname{Cone}\left(S^{1} \times Q\right) \times I^{2} \simeq Q$, then $\operatorname{Cone}\left(S^{1} \times Q\right) \times I^{2} \backslash\left\{\left(v_{1}, v_{2}\right)\right\}$ is contractible. But

$$
\begin{aligned}
& \operatorname{Cone}\left(S^{1} \times Q\right) \times I^{2} \backslash\left\{\left(v_{1}, v_{2}\right)\right\} \text { (homotopic) } \\
& \sim \operatorname{Cone}\left(S^{1}\right) \times I^{2} \backslash\left\{\left(v_{1}, v_{2}\right)\right\} \\
& \sim I^{4} \backslash\{\text { an interior point }\} \sim S^{3}
\end{aligned}
$$

which is not contractible. 


\section{REFERENCES}

[A] R. D. Anderson, Topological properties of the Hilbert cube and the infinite product of open intervals, Trans. Amer. Math. Soc. 126 (1967), 200-216. MR 34 \# 5045.

$\left[\mathrm{A}_{2}\right] \ldots, A$ characterization of apparent boundaries of the Hilbert cube, Notices Amer. Math. Soc. 16 (1969), 429. Abstract \#69T-G17.

[AB] R. D. Anderson and R. H. Bing, A complete elementary proof that Hilbert space is homeomorphic to the countable infinite product of lines, Bull. Amer. Math. Soc. 74 (1968), 771-792. MR 37 \# 5847.

[AK] R. D. Anderson and Nelly Kroonenberg, Open problems in infinite-dimensional topology, Math. Centre Tracts, No. 52, Mathematische Centrum, Amsterdam, 1974, pp. 141-175. MR 50 \#11247.

[BP] C. Bessaga and A. Pelczynski, Selected topics in infinite-dimensional topology, Monografie Mat., Vol. 58, PWN, Warsaw, 1975.

$\left[C_{1}\right]$ T. A. Chapman, Hilbert cube manifolds, Bull. Amer. Math. Soc. 76 (1970), 1326-1330. MR 44 \#3352.

$\left[\mathrm{C}_{2}\right] \_$, On the structure of Hilbert cube manifolds, Compositio Math. 24 (1972), 329-353. MR 46 \#4562.

[H] Michael Handel, The Bing staircase construction for Hilbert cube manifolds. (submitted).

$\left[\mathbf{H}_{2}\right] \ldots$ On certain sums of Hilbert cubes (submitted).

[K] Nelly Kroonenberg, Characterization of finite-dimensional Z-sets, Proc. Amer. Math. Soc. 43 (1974), 421-427. MR 48 \#12540.

[KU] K. Kuratowski, Topology, Vols. I, II, Academic Press, New York and London; PWN, Warsaw, 1966, 1968. MR 36 \# 840; 41 \#4467.

[NQS ${ }_{1}$ ] Sam B. Nadler, Jr., J. Quinn and Nick Stavrakas, Hyperspaces of compact convex sets. I, Bull. Polon. Acad. Sci. 23 (1975), 555-559.

$\left[\mathrm{NQS}_{2}\right]$ _ Hyperspaces of compact convex sets. II, Bull. Polon. Acad. Sci. (to appear).

[NQS $\left.{ }_{3}\right] \ldots$, Hyperspaces of compact convex sets, Pacific J. Math. (to appear).

[S] R. Sher, The union of two Hilbert cubes meeting in a Hilbert cube need not be a Hilbert cube (submitted).

$\left[W_{1}\right]$ J. E. West, Identifying Hilbert cubes: general methods and their application to hyperspaces by Schori and West, General Topology and Its Relation to Modern Analysis and Algebra, III (Proc. Third Prague Topological Sympos., 1971), Academia, Prague; Academic Press, New York, 1972, pp. 455-461.

$\left[\mathbf{W}_{2}\right] \ldots$, Infinite products which are Hilbert cubes, Trans. Amer. Math. Soc. 150 (1970), 1-25. MR 42 \# 1055.

[WK] Raymond Y. T. Wong and Nelly Kroonenberg, Unions of Hilbert cubes, Trans. Amer. Math. Soc. 211 (1975), 289-297.

Department of Mathematics, University of Grorgia, Athens, Grorgia 30601

Department of Mathematics, Univeristy of California, Santa barbara, California 93106 (Current address of R. Y. T. Wong)

Current address (J. Quinn): Department of Mathematics, University of North Carolina, Charlotte, North Carolina 28223 